
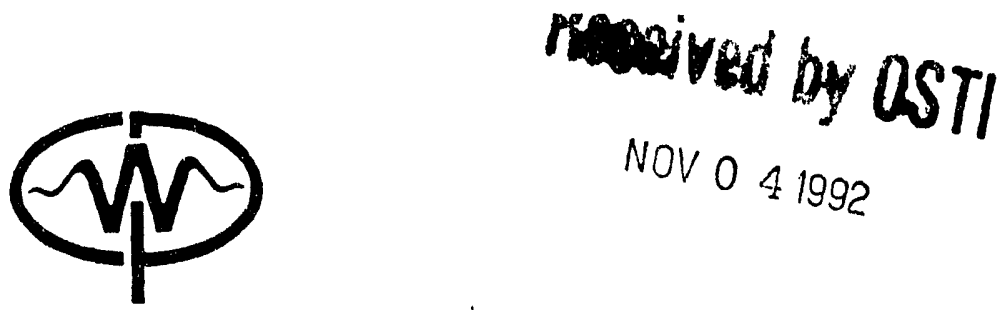

\title{
Migration Error in Transversely Isotropic Media with Linear Velocity Variation in Depth
}

\author{
Ken Larner and Jack K. Cohen
}

\section{DISCLAIMER}

\begin{abstract}
This report was prepared as an account of work sponsored by an agency of the United States
Government. Neither the United States Governmentored by an agency of the United States employees, makes any warranty, express or implied, nor any agency thereof, nor any of their bility for the accuracy, completeness, or usefulness of assumes any legal liability or responsiprocess disclosed, or represents that its use would of any information, apparatus, product, or ence herein to any specific commercial product, process, or manufacturer, or otherwise does not necessarily cocess, or service by trade name, trademark, mendation, or favoring by the United States Government or imply its endorsement, recomand opinions of authors expressed herein do United States Government or any agency thereof.
\end{abstract}

Center for Wave Fhenomena

Colorado School of Mines

Golden, Colorado 80401

$303 / 273-3557$ 


\title{
Migration error in transversely isotropic media with linear velocity variation in depth
}

\author{
Ken Larner and Jack K. Cohen
}

\begin{abstract}
Given the sensitivity of imaging accuracy to the velocity used in migration, migration founded (as in practice) on the erroneous assumption that a medium is isotropic can be expected to be inaccurate for steep reflectors. Here, we estimate errors in interpreted reflection time and lateral position as a function of reflector dip for transversely isotropic models in which the axis of symmetry is vertical and the medium velocity varies linearly with depth. We limit consideration to media in which ratios of the various elastic moduli are independent of depth.

Tests with reflector dips up to 120 degrees on a variety of anisotropic media show errors that are tens of wavelengths for dips beyond 90 degrees when the medium (unrealistically) is homogeneous. For a given anisotropy, the errors are smaller for inhomogeneous media; the larger the velocity gradient, the smaller the errors. For gradients that are representative of the subsurface, lateral-position errors tend to be minor for dips less than about 60 degrees, growing to iwo to five wavelengths as dip passes beyond 90 degrees.

These errors depend on reflector depth and average velocity to the reflector only through their ratio, i.e., migrated reflection time. Migration error, which is found to be unrelated to the ratio of horizontal to vertical velocity, is such that reflections with later migrated reflection times tend to be more severely overmigrated than are those with earlier ones.

Over a large range of dips, migration errors that arise when anisotropy is ignored but inhomogeneity is honored tend to be considerably smaller than those encountered when inhomogeneity is ignored in migrating data from isotropic, inhomogeneous media.
\end{abstract}




\section{INTRODUCTION}

Difficult and unreliable as it is to measure anisotropy in the field, and as infrequently as it is done, it is nevertheless currently recognized that the Earth's subsurface is anisotropic-often, considerably so. For example, a frequently used measure of anisotropy for p-waves, the ratio of velocity in the horizontal direction to that in the vertical direction, is commonly found to be 1.05 to 1.1 , and is often as large as 1.2 and higher (Seriff, 1986). For typical seismic wavelengths, the anisotropy may be either an intrinsic property of the rocks or the result of thin layering of different rock types. The distinction here is immaterial-the essential result is that waves travel with different speeds in different directions.

Given the general increase in wave speed with depth in the subsurface, reflections from steep interfaces-dips of 90 degrees and beyond-involve raypaths that sweep through a wide range of angles from vertical. Consequently, for inhomogeneous, anisotropic media, the energy propagates at different speeds due not only to variation in velocity with position but also to its variation with direction of propagation. Given the sensitivity of imaging accuracy to the velocity used in migration and given that migration, in practice, is founded on the assumption that the subsurface is isotropic, it is useful to analyze the positioning errors that arise from using migration algorithms that assume isotropy when the subsurface medium is not isotropic. This issue should be particularly relevant to the use of large-dip algorithms such as those that use turning waves (Hale, Hill, and Stefani, 1991) to image flanks of overhanging salt domes.

While anisotropy exists for both p-waves and s-waves, and s-wave anisotropy has been given the greater attention in the literature, most imaged reflection seismic data involve p-waves, and that is what we treat here. Moreover, although anisotropy can take on all manner of complexity, we assume the relatively simple, but plausible, form of transverse isotropy with a vertical axis of symmetry. That is, the velocity of plane waves (i.e., phase velocity) varies only with angle from the vertical; velocity is the same in all azimuthal directions.

Also, since the Earth's subsurface is not homogeneous (otherwise, among other things, turning waves would not exist), studies of migration error restricted to anisotropic models that are homogeneous can yield conclusions that, as we shall see, are greatly misleading. Again, actual subsurface inhomogeneity can be complicated and endlessly varied, so we limit consideration to a particularly simple form-media in which the pertinent elastic moduli vary only with depth $z$. Morecver, the allowed spatial variation will be such that ratios among the moduli remain independent of depth. Cervený (1989) refers to such media as factorized anisotropic inhomogeneous (FAI), and shows simplifications that arise when ray tracing in FAI media. Furthermore, following Shearer and Chapman (1988), we gain considerable efficiency in ray-trace modeling of traveltimes with our assumption that velocity variations are linear with depth. 
Here, we do numerical studies of errors in interpreted reflection time and lateral position as a function of reflector dip for models of the type described above. We treat only post-stack migration, and do so by considering only errors in the imaged position of sloping reflections for media with no lateral velocity variation. Those positioning errors are estimated from analysis of diffraction traveltime curves obtained by ray tracing; thus, they rely solely on traveltime information, ignoring amplitude and phase contributions to imaging.

Anisotropy and inhomogeneity can have a pronounced influence on the shape of diffraction curves, as evidenced by the comparison of diffraction curves for four models shown in Figure 1. All four curves pertain to a scatterer at depth $D=1500 \mathrm{~m}$, beneath midpoint $y=0$, in media having the same vertical average velocity from the surface to the reflector. They differ in that the different subsurface models represent the four combinations of homogeneous/inhomogeneous and isotropic/anisotropic media. Here and throughout this paper, the anisotropy is FAI transverse isotropy, with vertical axis of symmetry. The two inhomogeneous models (solid curves) involve linear $v(z)$, where $v$ represents any of the velocity-equivalents of the four elastic moduli describing $\mathrm{p}$-wave behavior in a transversely isotropic medium.

For those models, the vertical p-wave velocity is given by

$$
v(z)=v_{0}+k z,
$$

with the gradient $k=0.6 \mathrm{~s}^{-1}$, and the vertical-direction velocity at the surface $v_{0}$ is such that the vertical average velocity down to $D=1500 \mathrm{~m}$ is $3306 \mathrm{~m} / \mathrm{s}$, the value of vertical velocity for Levin's shale-limestone listed in Table 1. For the homogeneous, transversely isotropic model, the four elastic moduli $(A, C, F$, and $L$, in the notation of White [1983]) are those of the shale-limestone medium listed in Table 1. Actually listed in the table are velocities associated with the various moduli (i.e., $V_{C}=\sqrt{C / \rho}$ - where $\rho$ is bulk density-is the p-wave velocity in the vertical direction; $V_{A}$ is the p-wave velocity in the horizontal direction; $V_{L}$ is the s-wave velocity in the vertical direction; and $V_{F}$ is a velocity-like quantity associated with the elasticity modulus $F$ ). For the anisotropic model with linear $v(z)$, the moduli are such that their associated average velocities between the surface and the scatter at depth are equal to their constant-velocity counterparts in the homogeneous model.

In Figure 1, the curve for the homogeneous, isotropic model is a hyperbola, as expected, and the curves for the two inhomogeneous models are clearly non-hyperbolic, with inflection points at midpoints beyond which reflections pertain to turning waves (Hale et al., 1992). While the diffraction curve for the homogeneous, anisotropic model differs from that for the homogeneous, isotropic one, it is not evident from this figure whether or not the curve is hyperbolic. As it happens, it is not: a plot of the instantaneous slope of the $T^{2}$ versus $y^{2}$ line indicates that, while the $T^{2}$ versus $y^{2}$ is almost straight, it is not strictly so. Instantaneous "stacking" velocity based on the instantaneous slope of $T^{2}\left(y^{2}\right)$ increases with increasing midpoint value $y$, from a 


\begin{tabular}{|c|c|c|c|c|}
\hline Medium & $\begin{array}{c}\sqrt{C / \rho} \\
\mathrm{m} / \mathrm{s}\end{array}$ & $\begin{array}{c}\sqrt{A / \rho} \\
\mathrm{m} / \mathrm{s}\end{array}$ & $\begin{array}{c}\sqrt{F / \rho} \\
\mathrm{m} / \mathrm{s}\end{array}$ & $\begin{array}{c}\sqrt{L / \rho} \\
\mathrm{m} / \mathrm{s}\end{array}$ \\
\hline Berea sandstone & 4206 & 4210 & 1961 & 2664 \\
Shale-limestone & 3306 & 3721 & 2076 & 1819 \\
Cotton Valley shale & 4721 & 5320 & 3095 & 2890 \\
Pierre shale & 2202 & 2235 & 1803 & 969 \\
\hline
\end{tabular}

Table 1. Velocity-type quantities related to the four elastic moduli that are pertinent to $\mathrm{p}$-waves in transversely isotropic media. The four media listed are the same as those studied by Levin (1990).

value close to the velocity in the vertical direction at $y=0$ to one that is close to that for propagation in the horizontal direction as $y / D$ becomes large.

It is not obvious that the differences seen in the curves of Figure 1 would give rise to sizeable errors in migration when the wrong curve is used for the migration. As we shall see, however, for reflections from steep reflectors (i.e., for regions of the diffraction curves where $y$ is large), the migration errors can be large-even tens of wavelengths. As it happens, the largest errors arise when isotropy is assumed for media that are both anisotropic and homogeneous, rather than inhomogeneous.

\section{ERROR COMPUTATION}

The quality of a migration algorithm is usually assessed by applying the algorithm to synthetic and, ultimately, field data. Typical test data consist of reflections from plane-dipping reflectors, diffractions from point scatterers, and impulses. With data from plane reflectors, one measures the positicn of the migrated reflection relative to its known true location; with diffractions, one qualitatively assesses how well or poorly the diffractions collapse about the apex; and with impulses, one studies the shape of the impulse response.

Here, we are less interested in the quality of a particular migration algorithm than in errors that arise when the migration is based on an rrroneous assumption (e.g., isotropy) about the velocity model. In the context of th r rchhoff-summation view of migration, errors arise because the wrong diffraction . :e is used to do the migration: points on sloping reflections are migrated to the $\mathrm{r} g$ apex locations.

Consider migration of the schematic, zero-offset, sloping ction sl in in Figure 2. In migrating the portion of the reflection in the vicinity of point $(T, y)$, where $T$ is unmigrated reflection time, we first find the diffraction curve that is tangent to the reflection at $(T, y)$. If the velocity model is correct, migration will image the point $(T, y)$ at the correct migrated position $\left(T_{m}, y_{m}\right)$. Suppose, instead, that the 


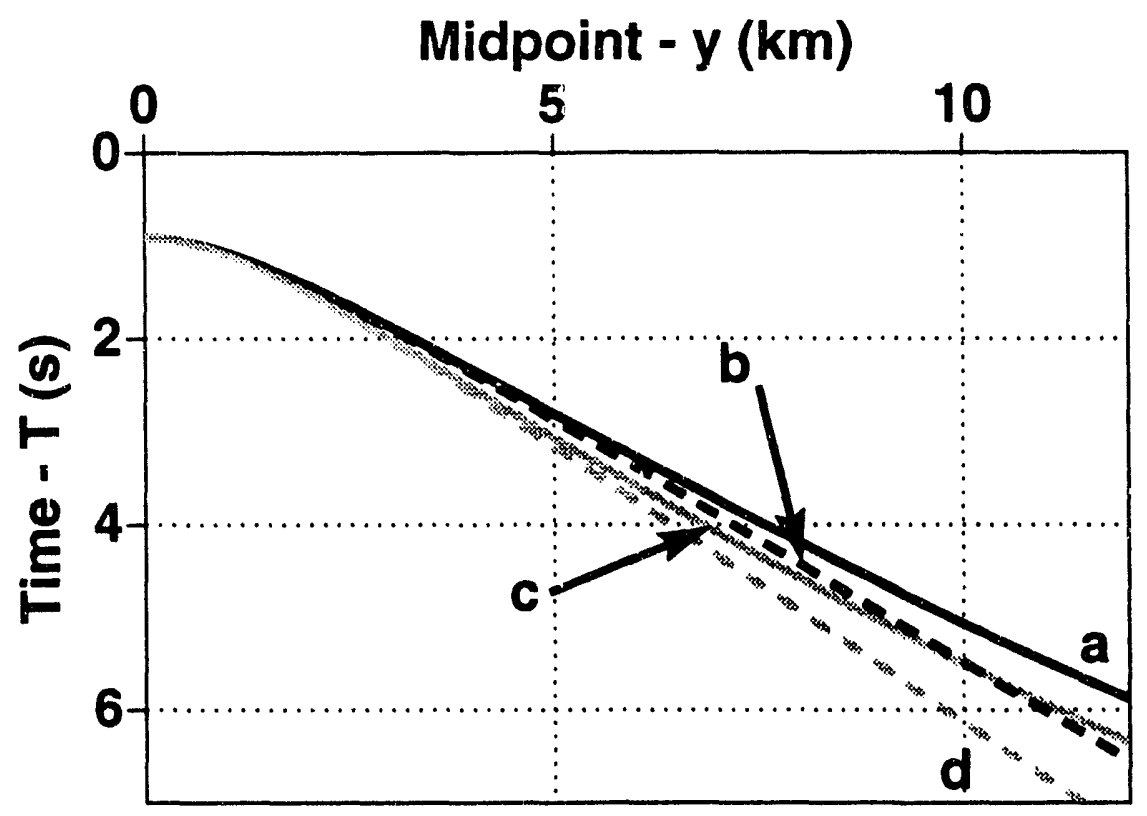

Fig. 1. Diffraction curves $T(y)$, where $T$ is two-way time between a surface source at midpoint $y$ and a scatterer at depth $D=1500 \mathrm{~m}$ beneath $y=0$, for four related media characterized by the same vertical average velocity. (a) transversely isotropic, with linear $v(z)$-black solid, (b) homogeneous, transversely isotropic-black dash, (c) isotropic with linear $v(z)$-gray solid, and (d) homogeneous, isotropic-gray dash. The latter curve is the only hyperbolic one.

wrong velocity model is used for the migration. Then, after migration, as depicted in Figure 3, the point $(T, y)$ goes to the erroneous position $\left(T_{e}, y_{e}\right)$ instead of to $\left(T_{m}, y_{m}\right)$.

Note that the point $\left(T_{e}, y_{e}\right)$ is at the apex of the erroneous diffraction curve that is tangent to both the correct diffraction curve and the reflection at the unmigrated position $(T, y)$. For the numerical estimates of migration error as a function of reflector dip, we do not actually compute reflections from plane-dipping reflectors. Instead, we work with just diffraction curves, recognizing that any point along a diffraction curve may be associated with a dipping reflector whose reflection is tangent to the diffraction curve at that point. The estimation of migration error involves three steps:

1. Compute diffraction curves associated with a buried point scatterer (such as those shown in Figure 1). 


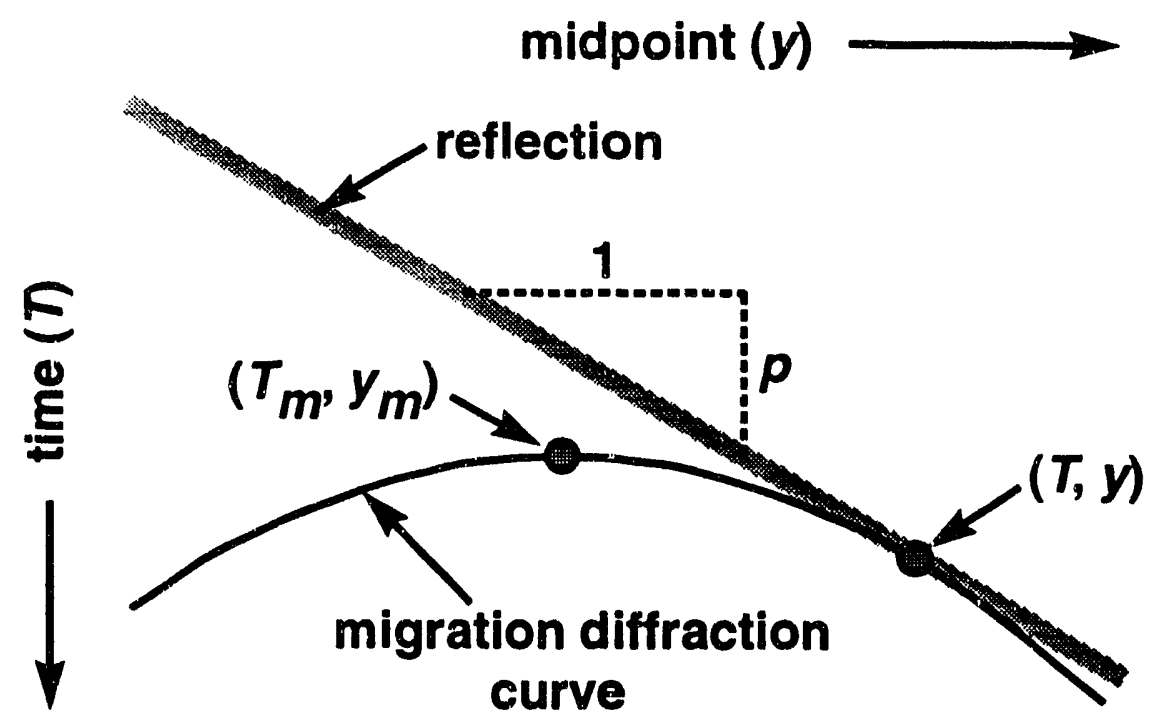

FIG. 2. Schematic time section showing the 1 elationship between the unmigrated position $(T, y)$ on a sloping reflection and the 'osition $\left(T_{m}, y_{m}\right)$ to which it should migrate. The migrated position is at the apex ot the diffraction curve that is tangent to the reflection (slope $=p$ ) at the unmigrated position.

2. Estimate the erronesus position $\left(T_{e}, y_{e}\right)$ to which any given point on the true diffraction curve for the anisotropic medium migrates when an algorithm that erroneously assumes isotropy is used for the migration.

3. Estimate the error in interpreted temporal and lateral position of a migrated reflection by determining the departures in position and time of the erroneously migrated point from the correctly migrated reflection.

\section{Computation of diffraction curves}

While traveltime computation in inhomogeneous media generally first requires computationally intensive numerical integration to obtain raypaths, such numericul integration can be averted for special classes of media. For example, in isotropic media with constant gradient in velocity, raypaths are circular so that two-point ray tracing and traveltime computation can be done analytically. Shearer and Chapman (1988) have developed an efficient method for ray tracing in the type of media considered here-FAI media with constant velocity gradient. For transversely isotropic media, the core of their result is the remarkable property that raypaths simply are scaled, rotated versions of the slowness curve, the curve that relates horizontal and vertical slowness at any point in the medium.

With this observation, Larner (1992) shows that, when the axis of symmetry for the transverse isotropy is parallel to the velocity gradient, two-point ray tracing can be done by solving a quadratic equation for $x_{1}^{2}$ as a function of $x_{3}^{2}$ followed by a 


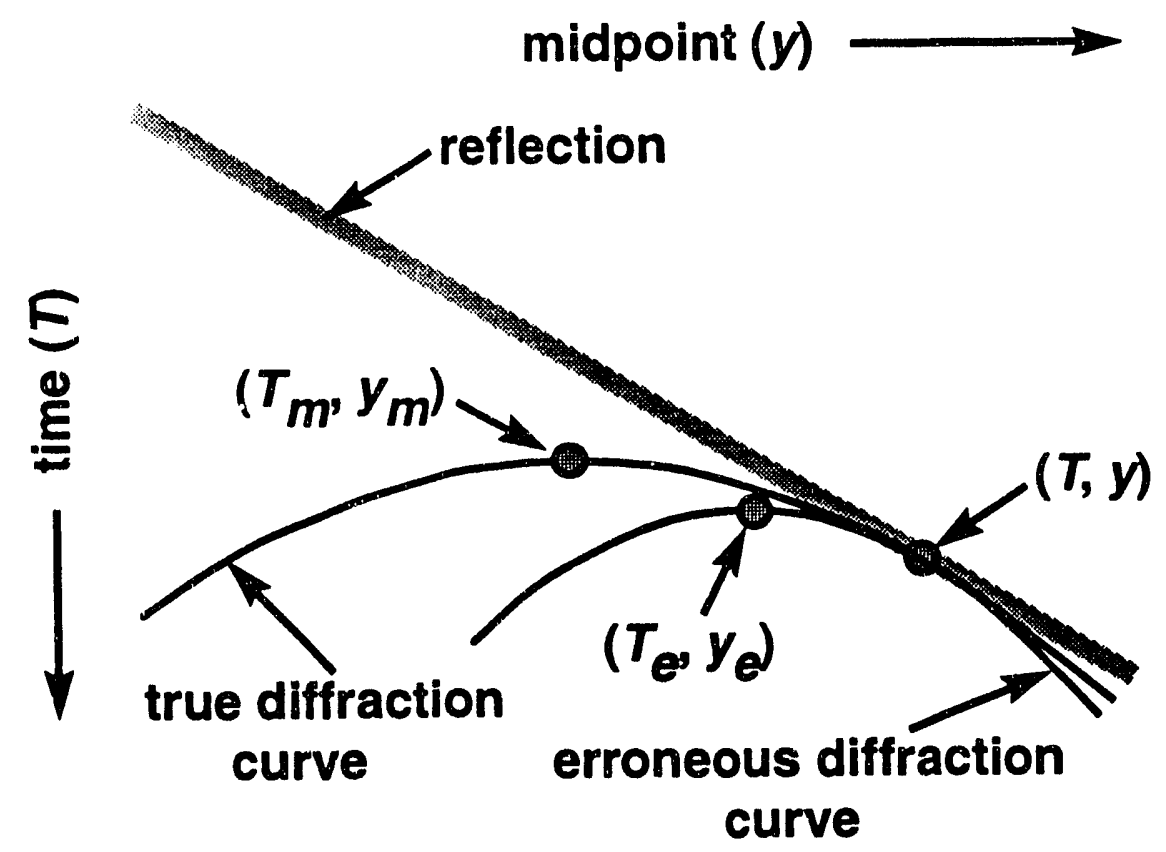

FIG. 3. Schematic time section showing the relationship between the unmigrated position $(T, y)$ on a sloping reflection, the position $\left(T_{m}, y_{m}\right)$ to which it should migrate, and the position $\left(T_{e}, y_{e}\right)$ at which it is actually imaged when the data are migrated with an erroneous velocity function.

secant search to determine the ray parameter $p_{1}$. As shown in Figure $4,\left(x_{1}, x_{3}\right)$ is position along the raypath, where $x_{3}$ is the coordinate in the gradient direction, with $x_{3}=0$ being the line along which the linear velocity function is zero, and $x_{1}$ is the coordinate in the orthogonal direction, such that $x_{1}=0$ at the ray's turning point. The coordinate $x_{3}$ is just a translated version of the depth $z$ obtained by rewriting equation (1) as

$$
v(z)=k\left(z+\frac{v_{0}}{k}\right)=k x_{3}
$$

where

$$
x_{3}=z+\frac{v_{0}}{k} \text {. }
$$

Note, in Figure 4, that reflectors such as the one shown, in general are not perpendicular to zero-offset raypaths when the medium is anisotropic. For anisotropic media, reflectors are orthogonal to the phase direction rather than the ray direction (Byun, 1984).

Different solutions of the quadratic equation for $x_{1}^{2}$ give raypaths for $\mathrm{p}$-waves and for sv-waves (Larner, 1992). Here, we are interested in only the p-wave solutions. Once the ray parameter is determined, numerical integration is still required to obtain traveltime; Cervený (1989), however, shows the form of the integrand to be particularly simple, and the integration thus efficient, for the particular type of 


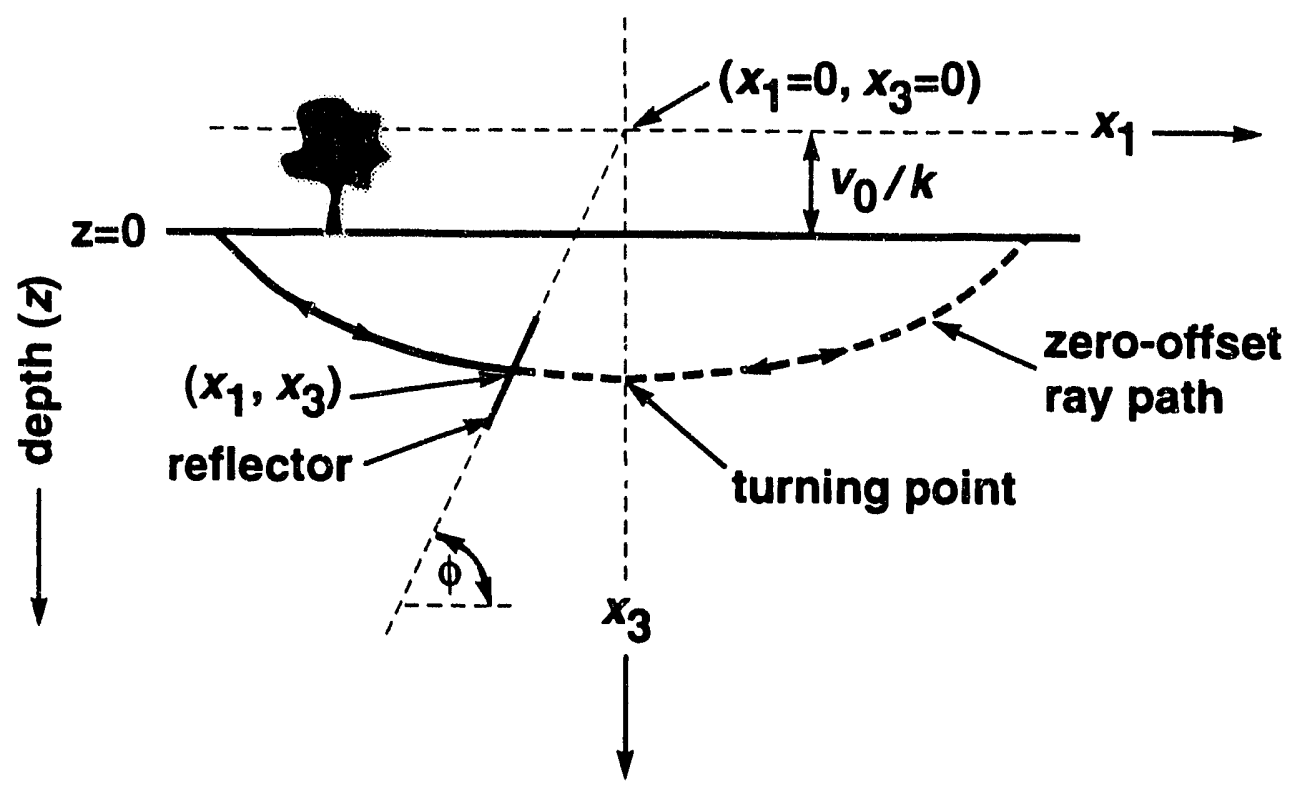

FIG. 4. Raypath in an FAI transversely isotropic, linear $v(z)$ half-space, shown in $\left(x_{1}, x_{3}\right)$ coordinates. The medium's surface, $z=0$, is equivalent to $x_{3}=v_{0} / k$. Also, $x_{1}=0$ at the turning point. If this is a zero-offset raypath, then the line shown with $\operatorname{dip} \phi$ represents a hypothetical reflector; the solid portion of the raypath pertains to a source-receiver location to the left of the reflector, and the dashed portion contains a turning ray that would image the underside of the reflector from the right. Note that the reflector is not perpendicular to the raypath except at the turning point.

anisotropic medium under consideration here. The procedure for computing $T\left(y_{j}\right)$ at uniformly sampled midpoint distances $y_{j}$ is described in Larner (1992). There, it is also shown that, for zero-offset rays in media of the type studied here, reflector dip $\phi$ at any point $\left(x_{1}, x_{3}\right)$ is given by

$$
\tan \phi=-\frac{x_{3}}{x_{1}}
$$

as indicated in Figure 4.

\section{Estimation of erroneous migrated position $\left(T_{e}, y_{e}\right)$}

As illustrated in Figure 3, to find the erroneous migrated position $\left(T_{e j}, y_{e j}\right)$ at which a point $\left(T_{j}, y_{j}\right)$ is imaged, we must find the diffraction curve that is associated with the migration-velocity model and is tangent to the true diffraction curve $T(y)$ at $\left(T_{j}, y_{j}\right)$. Specifically, the erroneous diffraction curve should have slope $p_{j}$ at $\left(T_{j}, y_{j}\right)$, where $p_{j}$ is computed as

$$
p_{j}=\frac{T_{j+1}-T_{j-1}}{y_{j+1}-y_{j-1}} .
$$

This would be no problem if the migration process were based on the assumption that the medium were homogeneous and isotropic. In that case, the migration diffraction 
curve would be a hyperbola, given by

$$
T_{j}^{2}=T_{e j}^{2}+\frac{4\left(y_{j}-y_{e j}\right)^{2}}{V_{m}^{2}},
$$

where $V_{m}$ is the migration velocity. Differentiating this equation gives the slope at $\left(T_{j}, y_{j}\right)$

$$
p_{j} \equiv \frac{d T_{j}}{d y_{j}}=\frac{4\left(y_{j}-y_{e j}\right)}{V_{m}^{2} T_{j}}
$$

or

$$
y_{e j}=y_{j}-\frac{p_{j} V_{m}^{2} T_{j}}{4}
$$

Also, the erroneously migrated time would be given by

$$
T_{e j}=T_{j} \cos \theta_{j}
$$

where

$$
\sin \theta_{j}=\frac{p_{j} V_{m}}{2}
$$

Assume instead that the data are migrated with an algorithm that honors vertical variation in velocity, but is founded on the assumption that the subsurface medium is isotropic (e.g., the phase-shift method of Gazdag [1978]). Then, the depth $z_{j}$ of the scatterer that would give rise to the migration diffraction curve with slope $p_{j}$ at $\left(T_{j}, y_{j}\right)$ is unknown at the outset, and hence, the migration velocity required to generate that migration diffraction curve is initially unknown.

To find that scatterer depth and associated velocity, we match the slopes of the true and migration diffraction curves at the point $\left(T_{j}, y_{j}\right)$, as follows. Let the erroneous, migration diffraction curve be given by $t(x)$, where, referring to Figure 3 ,

$$
x \equiv y-y_{e}
$$

is the migration distance. We assume that the velocity model used for migration is isotropic, with velocity given by equation (1). That is, the velocity at any depth $z$ is identical to the velocity in the vertical direction in the true, transversely isotropic medium. (Below, we shall modify this assumption somewhat.) For such a medium, raypaths are circles, and, using Slotnick (1986; equation (17), p. 237), the two-way time $t$ between a surface point at midpoint $y$ and a scatterer at depth $\left(y_{e}, z\right)$ is given by

$$
\begin{aligned}
t & =\frac{2}{k} \cosh ^{-1}\left(\frac{k^{2} x^{2}+v_{0}^{2}+v^{2}(z)}{2 v_{0} v(z)}\right) \\
& =\frac{2}{k} \cosh ^{-1}\left(\alpha x^{2}+\beta\right) \\
& =\frac{2}{k} \cosh ^{-1} \eta
\end{aligned}
$$


where

$$
\begin{aligned}
\alpha & \equiv \frac{k^{2}}{2 v_{0} v(z)} \\
\beta & \equiv \frac{v_{0}^{2}+v^{2}(z)}{2 v_{0} v(z)} \\
\eta & \equiv \alpha x^{2}+\beta=\cosh \frac{k t}{2} .
\end{aligned}
$$

Differentiating equation (6) gives the slope of the diffraction curve

$$
p \equiv \frac{d t}{d x}=\frac{4 \alpha x}{k \sqrt{\eta^{2}-1}}
$$

Given measurements of $T_{j}(=t)$ and $p_{j}$, and assuming that the constants $v_{0}$ and $k$ in equation (1) are known, we eliminate $x$ from equations (7) and (8). The result is a quadratic equation for $v\left(z_{j}\right)$, whose appropriate solution is

$$
v\left(z_{j}\right)=\frac{v_{0}\left(\cosh \delta+\sinh \delta \sqrt{1-g^{2}}\right)}{1+g^{2} \sinh ^{2} \delta}
$$

where

$$
\begin{aligned}
\delta & =\frac{k t_{j}}{2} \\
g & =\frac{p_{j} v_{0}}{2} .
\end{aligned}
$$

Once $v\left(z_{j}\right)$ has been determined, the depth $z_{j}$ is obtained from equation (1), $x_{j}$ from equation (8), and $y_{e j}$ from equation (5). Finally, the vertical reflection time to the scatterer is given by

$$
T_{e j}=2 \int_{0}^{z_{j}} \frac{d \sigma}{v_{0}+k \sigma}=\frac{2}{k} \log \frac{v\left(z_{j}\right)}{v_{0}} .
$$

\section{Estimation of migration error}

We have just seen how the erroneous migrated position $\left(T_{e j}, y_{e j}\right)$ is computed. In addition, the correct position $\left(T_{m}, y_{m}\right)$ is known to be just the apex of the diffraction curve $\left(T_{j}, y_{j}\right)$ obtained by ray tracing in the FAI transversely isotropic medium. At first thought, it might seem from Figure 3 that the sought-after errors in migrated time and position are just $\left(T_{e}-T_{m}\right)$ and $\left(y_{e}-y_{m}\right)$, respectively (from here on, the subscript $j$ is dropped). While in a sense that is true, such measures will not suffice for assessing errors in the positions of reflections that confront interpreters. Interpreters rarely identify how individual points in data move when data are migrated. Instead, 
they generally focus attention on reflections that, if not planar, are often locally approximately so.

Consider the sloping reflections in the schematic, migrated zero-offset section shown in Figure 5a. In practice, the incorrectly migrated reflection through migrated point $\left(T_{e}, y_{e}\right)$ would be approximately parallel to the correctly migrated reflection through $\left(T_{m}, y_{m}\right)$, as depicted in the figure. The quantities $\Delta y$ and $\Delta T$ shown in the figure are the measures of error that would be apparent to the interpreter. Note that given $\left(T_{e}, y_{e}\right)$ and $\left(T_{m}, y_{m}\right), \Delta y$ and $\Delta T$ are dependent on the slope $p_{m}$ of the migrated reflection. That is, one has to know, or estimate, $p_{m}$ in order to compute $\Delta y$ and $\Delta T$. This fact becomes obvious when Figures $5 \mathrm{a}$ and $5 \mathrm{~b}$ are compared. Figure $5 \mathrm{~b}$ depicts a situation where the apex positions $\left(T_{e}, y_{e}\right)$ and $\left(T_{m}, y_{m}\right)$ are identical to those in Figure 5a, but the slopes of the reflections differ greatly in the two figures. Figure 5a might pertain to a reflector dip that is less than 90 degrees, and Figure 5b to one that exceeds 90 degrees. As a result, the migration errors $\Delta y$ and $\Delta T$ differ considerably in the two figures.

The dependence of $\Delta y$ and $\Delta T$ on the slope $p_{m}$ is readily seen in the geometry of Figure 5. We have

$$
\begin{aligned}
\Delta y & =\left(y_{e}-y_{m}\right)-\frac{T_{e}-T_{m}}{p_{m}} \\
\Delta T & =-p_{m} \Delta y .
\end{aligned}
$$

So, these two interrelated measures of migration error can be fully estimated once we know the slope $p_{m}$ of the correctly migrated reflections. That slope is readily computed from knowledge of $v(z)$ and the reflector dip $\phi$. Such a reflector is depicted in Figure 6. Also shown are vertical "paths" from two surface points separated by the distance $\Delta y$ down to the reflector. Migrated reflection time at the two surface points is just the two-way time along these vertical paths. Locally, the migrated reflection slope is taken as constant, given by

$$
\begin{aligned}
p_{m} & \equiv \frac{\Delta T_{m}}{\Delta y} \approx \frac{d T_{m}}{d y} \\
& =\frac{d T_{m}}{d z} \frac{d z}{d y}=\tan \phi \frac{d T_{m}}{d z}
\end{aligned}
$$

But, for any $v(z)$ medium,

$$
T_{m}=2 \int_{0}^{z} \frac{d \sigma}{v(\sigma)}
$$

so

$$
\frac{d T_{m}}{d z}=\frac{2}{v(z)}
$$



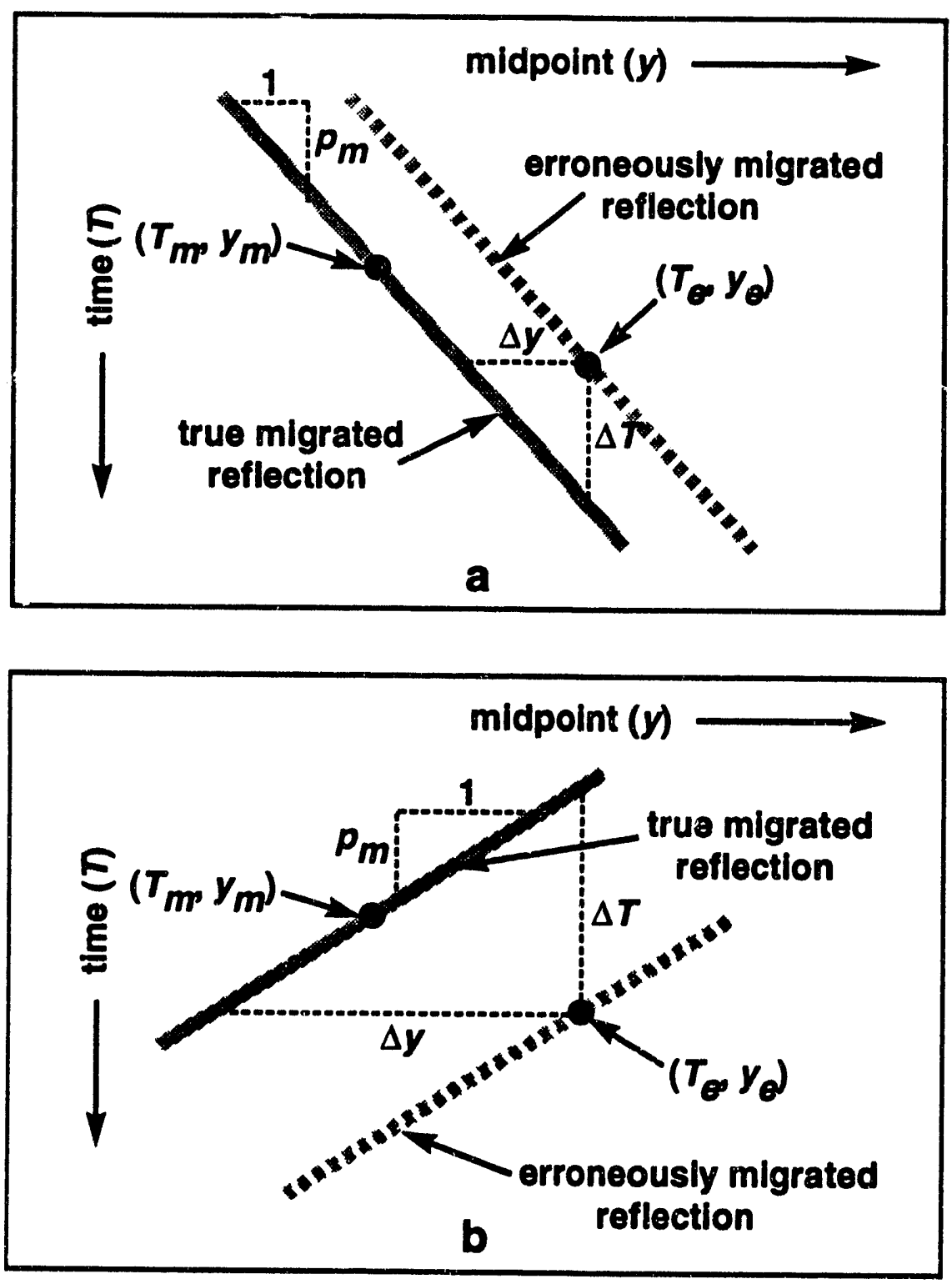

FIG. 5. Schematic time sections showing the erroneously migrated (dashed) and correctly migrated (solid) reflections through the erroneously and correctly imaged positions $\left(T_{e}, y_{e}\right)$ and $\left(T_{m}, y_{m}\right)$. The lateral error in imaged reflection position is $\Delta y$, and the time error is $\Delta T$. Figures $5 \mathrm{a}$ and $5 \mathrm{~b}$ differ only in that the slope $p_{m}$ of the migrated reflection differs in the two cases. 


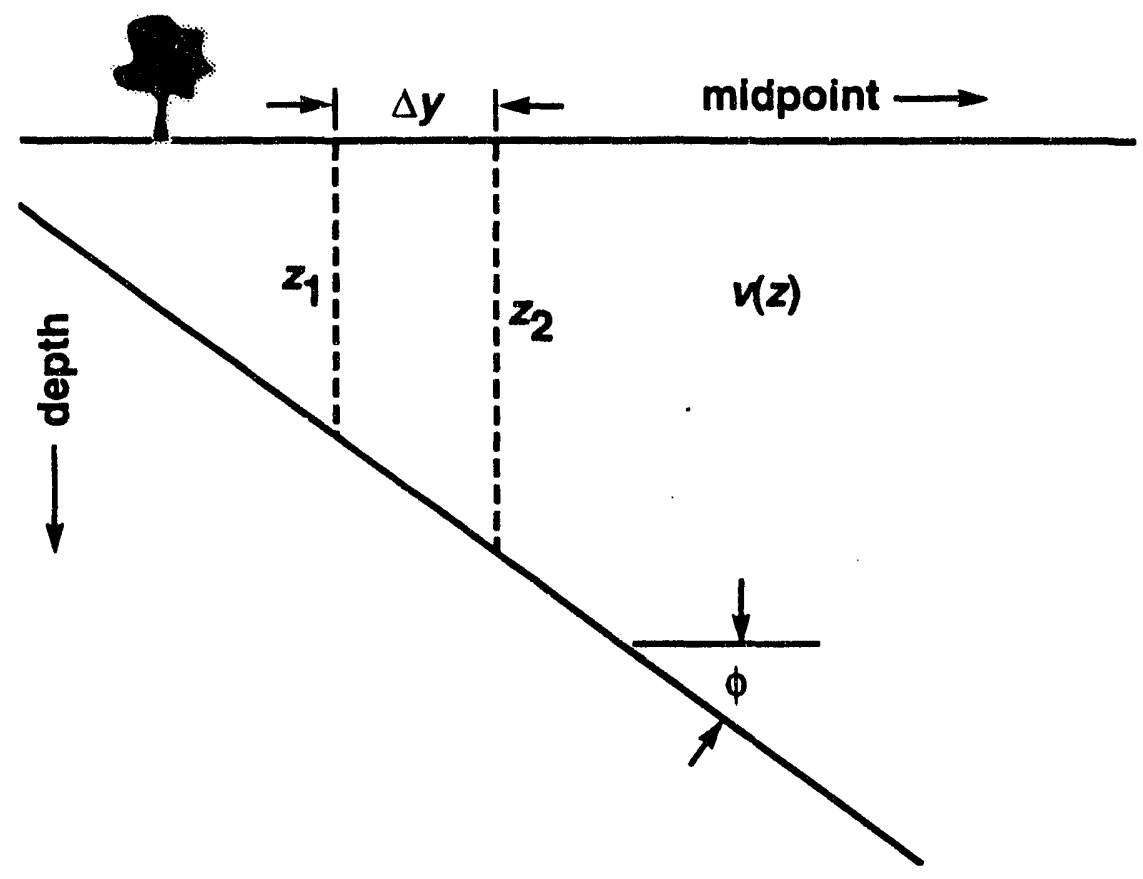

FIG. 6. Schematic depth section showing a dipping reflector beneath a $v(z)$ medium. Migrated reflection times at two neighboring points on the surface are simply two-way times along the vertical paths.

from which we get

$$
p_{m}=\frac{2 \tan \phi}{v(z)} .
$$

Note that this result, which is familiar for homogeneous media, holds for any $v(z)$ medium, even a generally anisotropic one. For our error studies, then, we have all the information required to compute the migration errors $\Delta y$ and $\Delta T$ once we can associate any point $\left(T_{j}, y_{j}\right)$ along the true diffraction curve (for a scatterer at depth $z$ ) with a reflector dip $\phi_{j}$. That dip is available from the ray-tracing result, equation (3).

\section{WHAT FORM OF VELOCITY TO USE FOR MIGRATION}

Reiterating, our estimates of migration error come from relating the true diffraction curve (i.e., that for an FAI transversely isotropic medium with linear $v(z)$ depeidence) to the diffraction curve associated with the time-migration process used. Almost universally in practice, that migration process is based on the assumption that the subsurface is isotropic and vertically inhomogeneous. As is known, if the medium were homogeneous and isotropic, no issue would arise as to which form of velocity to use in the migration - migration velocity $=$ root-mean-square $(\mathrm{rms})$ velocity = stacking velocity (assuming horizontal reflectors) = medium velocity. Equally well known, for vertically inhomogeneous, isotropic media, stacking velocity (obtained by 
$T^{2}-X^{2}$ analysis over a finite spreadlength $X$ ) exceeds rms velocity, approaching it as the spreadlength approaches zero. When the medium is anisotropic (even when it is homogeneous), the situation becomes more complex.

Levin (1979) has shown that, in a transversely isotropic medium with vertical axis of symmetry, stacking velocity for p-wave reflections from horizontal interfaces differs from the vertical velocity, even in the limit as spreadlength $X$ approaches zero. The limiting stacking velocity can be larger or smaller than the vertical velocity, depending on ratios among the four pertinent elastic moduli. Moreover, Thomsen (1986) shows that, for so-called weak anisotropy, the relationship between these two types of velocity is totally independent of the ratio of horizontal-direction velocity to vertical-direction velocity, $V_{A} / V_{C}$-the most commonly quoted measure of degree of anisotropy. As we shall see, this same discrepancy between zero-offset-limit stacking velocity and vertical velocity in homogeneous media carries over into a difference between zero-offset-limit stacking velocity and vertical $r m s$ velocity for inhomogeneous media.

Given this complexity, for studies of migration error and, indeed, when doing migration in practice, which form of velocity should we use-vertical rms velocity, stacking velocity based on finite spreadlength, or the limiting stacking velocity as offset approaches zero?

Figure 7 shows computed lateral position errors $\Delta y$ for reflector dip ranging from 0 to 120 degrees, for four different choices of migration velocity. Results are shown for the shale-limestone and Cotton Valley shale tabulated in Table 1 (for all but the shale-limestone, the quantities shown in Table 1 are computed from the parameters of Thomsen [1986]). The media treated in Figure 7 have linear velocity increase with depth, with vertical average velocity matching the $V_{C}=\sqrt{C / \rho}$ values in Table 1 (e.g., the vertical average velocity for the inhomogeneous shale-limestone medium is 3306 $\mathrm{m} / \mathrm{s})$. For these tests, the reflector depth is $1500 \mathrm{~m}$, and the gradient $k=0.6 \mathrm{~s}^{-1}$ in the vertical-velocity expression $v(z)=v_{0}+k z$.

Not surprising, as seen in Figure 7, the position errors depend on the velocity function used for the migration. For both the shale-limestone and Cotton Valley shale, migration errors are smallest for the migration-velocity function $v(z)$ that is based on stacking velocity computed when the spreadlength is comparable to reflector depth - a satisfying result since, in practice, velocity is most often estimated in this way. From the figure, the poorest choice of velocity function for migration is that based on the vertical rms velocity, such as might be obtained from sonic-log data.

Perhaps most striking in the Figure 7 is the dramatically anomalous error behavior for the Cotton Valley shale when the migration velocity is based on the rms velocity. For the shale-limestone, errors for stacking velocity approach those for rms velocity as the spreadlength shrinks to zero. Such is not the case for the Cotton Valley shale. This behavior for migration error is consistent with Thomsen's (1986) predictions that the small spreadlength stacking velocity for transversely isotropic media can depart significantly from vertical velocity. 

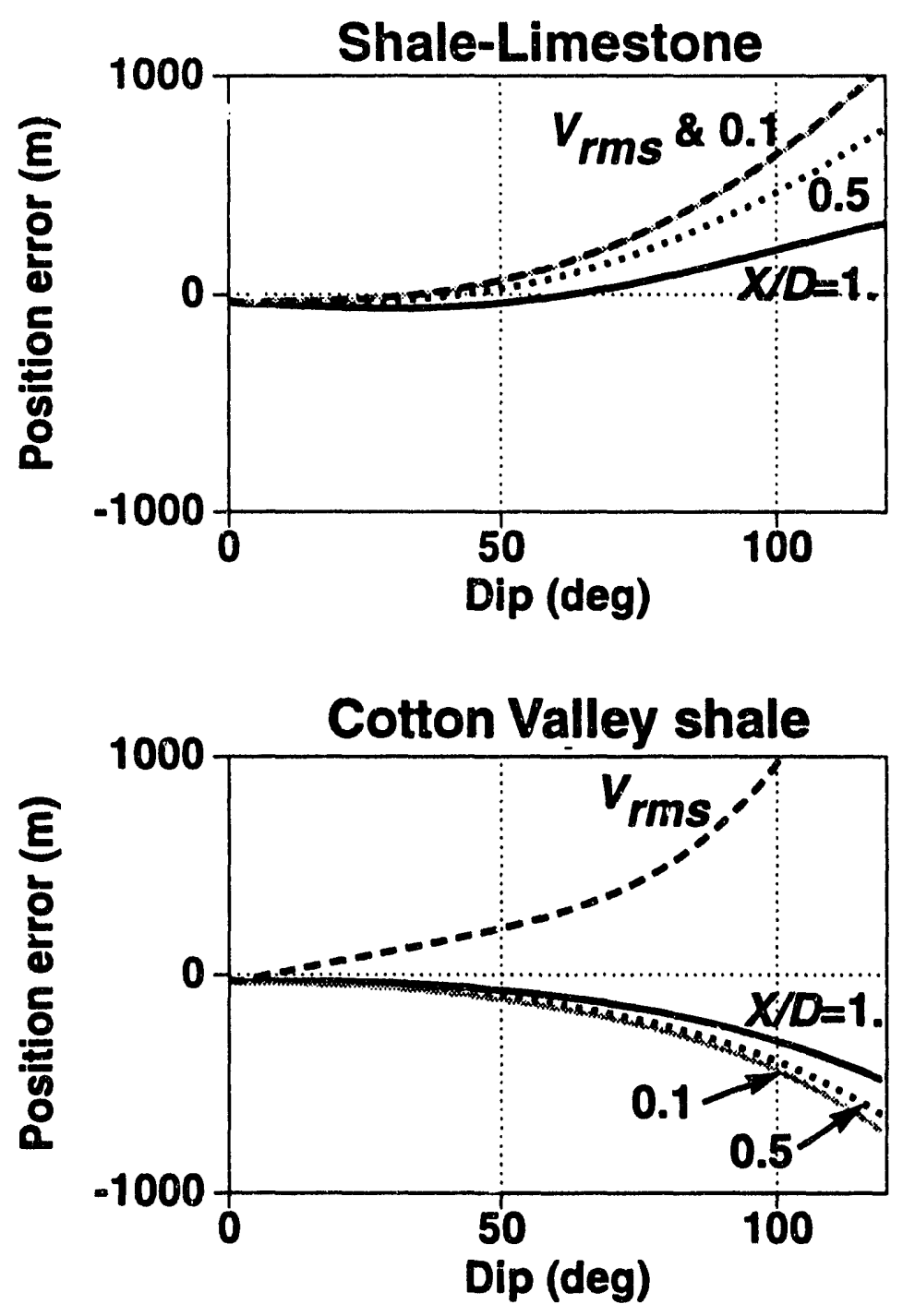

Fig. 7. Position error $\Delta y$ versus reflector dip for shale-limestone (top) and Cotton Valley shale (bottom). For each, the reflector depth is $D=1500 \mathrm{~m}$ and the vertical velocity gradient is $k=0.6 \mathrm{~s}^{-1}$. For the dashed curve, the $v(z)$ velocity function used for migration is derived from the vertical rms velocity to the reflector. For the other three curves, the $v(z)$ velocity function used for migration is derived from the stacking velocity to a horizontal reflector at depth $D$, with different choices of ratio of spreadlength to depth $X / D$ used in the stacking-velocity computation. 


\begin{tabular}{|c|c|c|c|c|c|}
\hline \multirow{2}{*}{ Medium } & $V_{A} / V_{C}$ & \multicolumn{4}{|c|}{$V_{\text {stack }} / V_{\text {rms }}$} \\
\cline { 3 - 6 } & & $X / D=1.0$ & $X / D=0.5$ & $X / D=0.1$ & Thomsen \\
\hline Berea sandstone & 1.001 & 1.01 & 1.02 & 1.02 & 1.02 \\
Shale-limestone & 1.126 & 1.06 & 1.02 & 1.00 & 1.00 \\
Cotton Valley shale & 1.127 & 1.16 & 1.18 & 1.19 & 1.19 \\
Pierre shale & 1.015 & 1.04 & 1.05 & 1.06 & 1.06 \\
\hline
\end{tabular}

Table 2. For the four media treated in this study, columns 3-5 list the ratio of stacking velocity to vertical $\mathrm{rms}$ velocity for three values of the ratio of the spreadlength to reflector depth. For comparison, column 6 contains the ratio of zero-offset stacking velocity to $\mathrm{rms}$ velocity predicted by Thomsen (1986). Column 2 lists the ratio of horizontal to vertical medium velocity - the most frequently quoted measure of degree of anisotropy.

Table 2 gives a summary of the ratio $V_{\text {stack }} / V_{\text {rms }}$ measured in our studies with transversely isotropic, linear $v(z)$ media. For comparison are shown Thomsen's predictions, which were derived for homogeneous transversely isotropic media. The equality of values in columns 5 and 6 of the table shows that Thomsen's predictions extend to inhomogeneous media. Moreover, also in agreement with Thomsen's predictions, note the considerable differences between the values in column 6 for the shale-limestone and Cotton Valley shale despite the fact that the ratios of horizontal to vertical velocity for the two media (column 2) are nearly ident :al.

In summary, based on the curves shown in Figure 7, along with those for the other media studied (Berea sandstone and Pierre shale, not shown here), the migrationvelocity function that is derived from stacking velocity computed when the spreadlength is comparable to reflector depth yields the smallest of the errors. Thus, both in accord with these results and mimicking common practice, the velocity function that we use for all the migration-error tests below is based on stacking velocity (for horizontal reflectors) with $X / D=1$, and the migration action that we simulate fully takes into account velocity variation with depth.

\section{MIGRATION-ERROR RESULTS}

\section{Anisotropy versus inhomogeneity}

Figure 8 shows position error $\Delta y$ (top) and time error $\Delta T$ (bottom) as a function of reflector dip ranging from 0 to 120 degrees for three different models, all of which have properties related to those of the transversely isotropic shale-limestone listed in Table 1. For all three curves, the reflector depth is $1500 \mathrm{~m}$. The solid gray curves pertain to a homogeneous medium with just the properties listed in Table 1 . The solid black curves are for a $v(z)=v_{0}+k z$ medium with gradient $k=0.6 \mathrm{~s}^{-1}$, and with $v_{0}$ 
such that the vertical average velocity to the reflector is $3306 \mathrm{~m} / \mathrm{s}$. Consider, first, just the position errors. The gray curve exhibits large error for dips greater than about 60 degrees. Clearly, if we limited our analysis to just homogeneous media, we would conclude that failure to take anisotropy into account would lead to migration errors that are intoicrably large for steep reflectors. However, we find that the combination of anisotropy and velocity variation with depth (solid black curve) leads to much more acceptable errors-even for dips as large as 120 degrees. As we shall see below, in some sense the shale-limestone is the most extreme of the four types of media studied here. For the other inedia, the errors, even for the homogeneousi models, are not so large as those shown here. In all cases, nevertheless, errors are smaller for the inhomogeneous models than for their (unrealistic) homogeneous counterparts.

Now, consider the dotted curve in the top part of the figure. This curve pertains to an isotropic medium with $v(z)$ identical to the vertical-velocity function in the transversely isotropic shale-limestone. If we were to migrate data from such a medium with an algorithm that honors the $v(z)$ behavior, such as the phase-shifi method of Gazdag (1978) as extended for turning :vaves by Hale, et al. (1992), then we would get near-zero error for all dips. The dotted curve, however, simulates errors that would arise if the data were migrated with a Kirchhoff-type time-migration algorithm (Schneider, 1978) that sinplistically uses a hyperbolic diffraction curve based on the stacking velocity. Such a limited algorithm is known to yield unacceptable errors for steep reflectors; the dotted curve, then, shows the size of error that is corrected when a phase-shift-type migration approach is used.

Stated differently; the dotted curve gives the errors when inhomogeneity is not properly treated in the migration of an inhomogeneous, isotropic medium, while the gray curve gives the errors when anisotropy is not taken into account in the migration of a homogeneous, transversely isotropic medium with the shale-limestone properties. Significantly, for dip less than about 60 degrees, anisotropy is the considerably less serious issue.

Comforting as it may be that errors are not so large when the medium is both inhomogeneous and anisotropic, we should still note that the errors for the poorly migrated isotropic medium are correctable (with, for example, a phase-shift algorithm), whereas those for the anisotropic medium would require that we have adequate information sbout the four pertinent elastic moduli of the medium and that we use an imaging algorithm that takes the anisotropy into account.

The time-error curves in the lower part of Figure 8 tell a comparable story, but they also show what appears to be a disturbing instability near 90-degree dip. Actually, the behavior is not so troubling as it may appear. For a plane reflector near vertical, a huge error in reflection time would not be noticeable to the interpreter since the temporal period of migrated reflections becomes large without bounds as dip approaches 90 degrees. Similarly, a seemingly large error $\Delta y$ in lateral position is inconsequential for small dip since the apparent wavelength in the lateral direction becomes large as dip approaches zero. Whether it be time error or position error, 

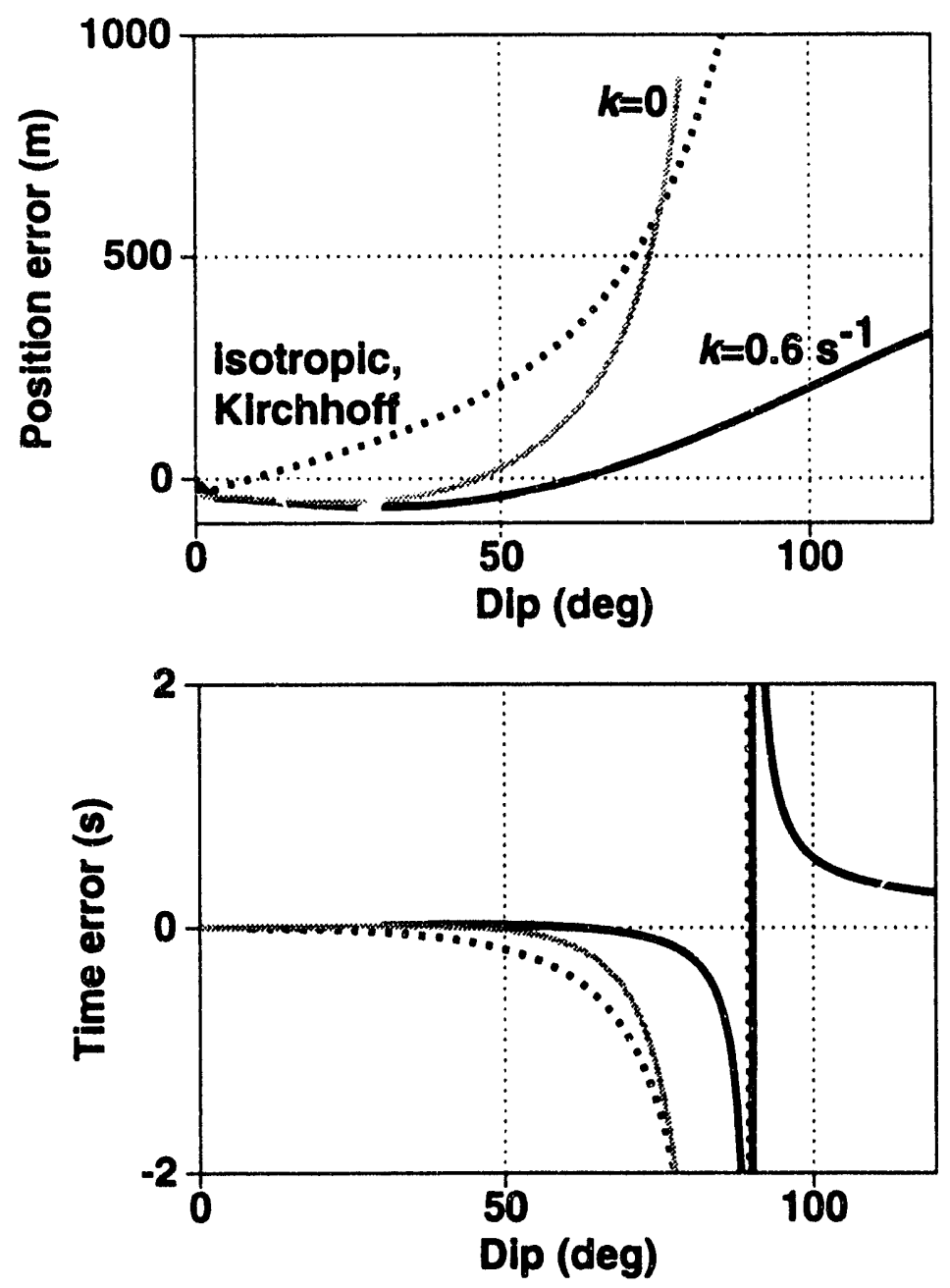

FIG. 8. Position error $\Delta y$ (top) and time error $\Delta T$ (bottom) versus reflecior dip for three different cases related to the transversely isotropic shale-limestone. Solid black: The actual medium is inhomogeneous with $v(z)=v_{0}+0.6 z$ and anisotropic; the inhomogeneity is honored in the migration, and the plotted errors are due to neglect of the anisotropy. Gray: The medium is the anisotropic shale-limestone, but now velocity is constant; the plotted errors are again due to the neglect of anisotropy in the migration. Dotted: Now the actual medium is isotropic but inhomogeneous, with linear $v(z)$, as above; the plotted errors are due to neglect of the inhomogeneity (e.g., migration is done with a Kirchhoff-type algorithm that uses hyperbolic diffraction curves based upon stacking velocity). 
the interpreter will be concerned only with errors that are large relative to a period, or wavelength, as the case may be. For that reason, henceforth, we shall study errors normalized, as follows, so that they are expressed in terms of multiples of the dominant period and wavelength in the migrated data.

Earlier, we saw that migration increases the slope of a reflection from $p$ before migration to $p_{m}=2 \tan \phi / v(z)$, afterward. Migration also lowers frequencies such that horizontal wavelength $\lambda$ is preserved. Consequently,

$$
\frac{1}{\lambda}=f_{m} p_{m}=f p
$$

where $f$ is frequency in the unmigrated reflection wavelet, and $f_{m}$ is the frequency after migration.

Subsequent position-error curves in this paper are plotted in terms of normalized values given by

$$
\Delta \tilde{y}=\frac{\Delta y}{\lambda_{d}}
$$

where, from equation (14),

$$
\lambda_{d}=\frac{1}{f_{d} p}
$$

is the horizontal wavelength after migration, corresponding to some assumed dominant frequency $f_{d}$ in the unmigrated reflections.

If we similarly normalize time errors such that

$$
\begin{aligned}
\Delta \tilde{T} & =\frac{\Delta T}{\tau_{d}} \\
\tau_{d} & =p_{m} \lambda_{d},
\end{aligned}
$$

from equation (12) it follows that, simply,

$$
\Delta \tilde{T}=-\Delta \tilde{y}
$$

Note, that since $p_{m}<0$ for dips exceeding 90 degrees, $\tau_{d}$ as defined in equation (17) is also negative for those large dips. This unusual choice, rather than defining $\tau_{d}$ to be always positive, is a convenience that ensures that the normalized time error is continuous at 90-degree dip-indeed that it is just the negative of the normalized position error. This being the case, henceforth we show all migration errors as normalized position errors $\Delta \tilde{y}$.

Figure 9 shows the normalized position error $\Delta \tilde{y}$ corresponding to the error $\Delta y$ in Figure 8. For the normalization here and in subsequent figures, the dominant frequency is taken as $30 \mathrm{~Hz}$.

While the errors for the homogeneous shale-limestone can be very large for steep reflectors (more than ten wavelengths for dips exceeding about 60 degrees), errors 
for the inhomogeneous counte:part are about two wavelengths for vertical reflectors, and just four wavelengths at 120-degree dip. Again, these errors are much smaller than the errors for isotropic media that are corrected when a phase-shift algorithm, as opposed to one that involves hyperbolic diffractions, is used for migration.

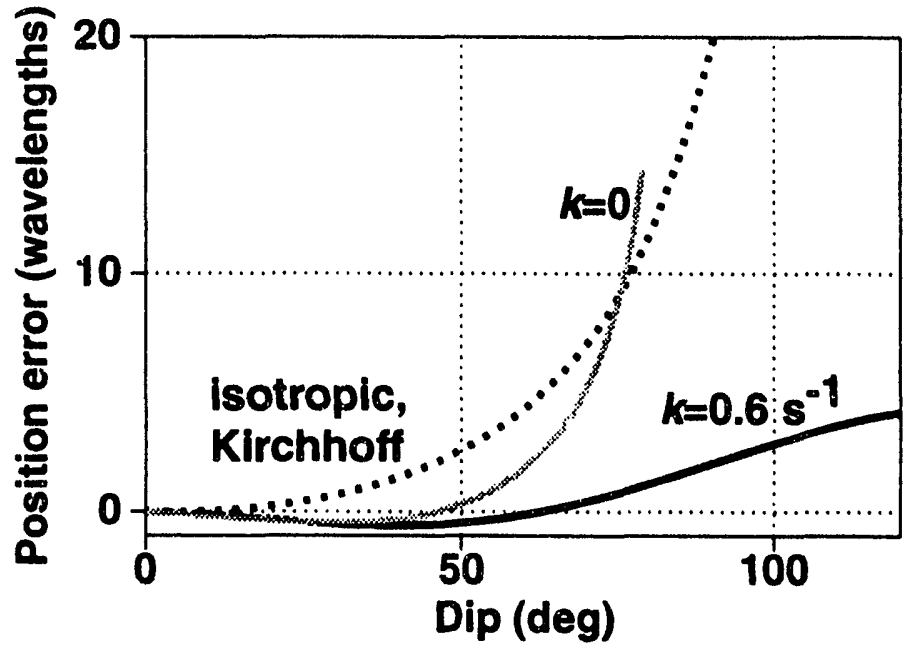

FIG. 9. Same as the top part of Figure 8 except that the pusition errors are now the normalized quantities $\Delta \tilde{y}$, expressed in multiples of lateral wavelengths corresponding to unmigrated reflections with dominant frequency $30 \mathrm{~Hz}$.

\section{Dependence on velocity gradient}

With reflector depth fixed at $1500 \mathrm{~m}$ and the vertical average velocity at $3306 \mathrm{~m} / \mathrm{s}$, Figure 10 shows the dependence of normalized position errors on the velocity gradient, $k$, for the shale-limestone, and Figure 11 shows the normalized position errors for the four media listed in Table 1. For all four media, the failure to take anisotropy into account in migration is a less serious issue when the medium is inhomogeneous than if it were homogeneous, but the difference is truly significant only for the shalelimestone.

Not surprising, errors in most cases tend to be larger for larger reflector dip. Of the four media, the Berea sandstone is most weakly anisotropic, and gives errors that are least influenced by the inhomogeneity. The shale-limestone is anomalous in that the position errors for larger dips are positive, whereas errors for the other media are negative. Thus, steep reflectors in the shale-limestone are under-migrated when anisotropy is not taken into account in the migration algorithm, while those in the other media are over-migrated. Intuition might lead one to predict that the presence of anisotropy would cause data to be under-migrated since isotropy-based migration algorithms fail to adapt to the higher propagation speeds that arise for waves that 


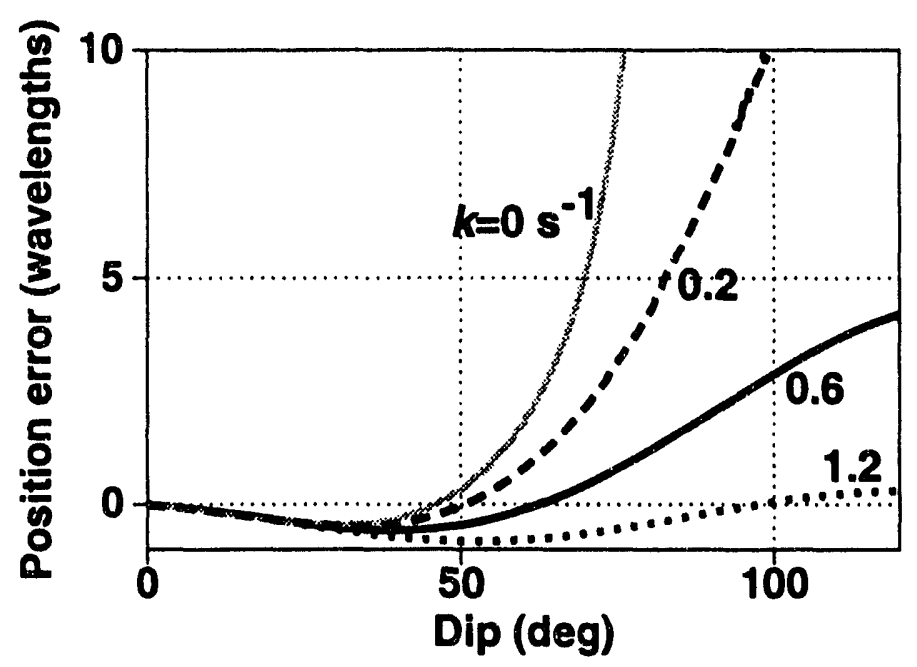

Fig. 10. Normalized position errors (at $30 \mathrm{~Hz}$ ) as a function of reflector dip for the shale-limestone, with velocity gradients $k=0.0,0.2,0.6$, and $1.2 \mathrm{~s}^{-1}$. All models have the same vertical average velocity, $3306 \mathrm{~m} / \mathrm{s}$, and the reflector depth is $1500 \mathrm{~m}$ in all cases. In all cases the medium is inhomogeneous and anisotropic, and the inhomogeneity is honored in the migration. The plotted errors are due to neglect of the anisotropy in the migration. Each curve shows the errors for the indicated velocity gradient. 

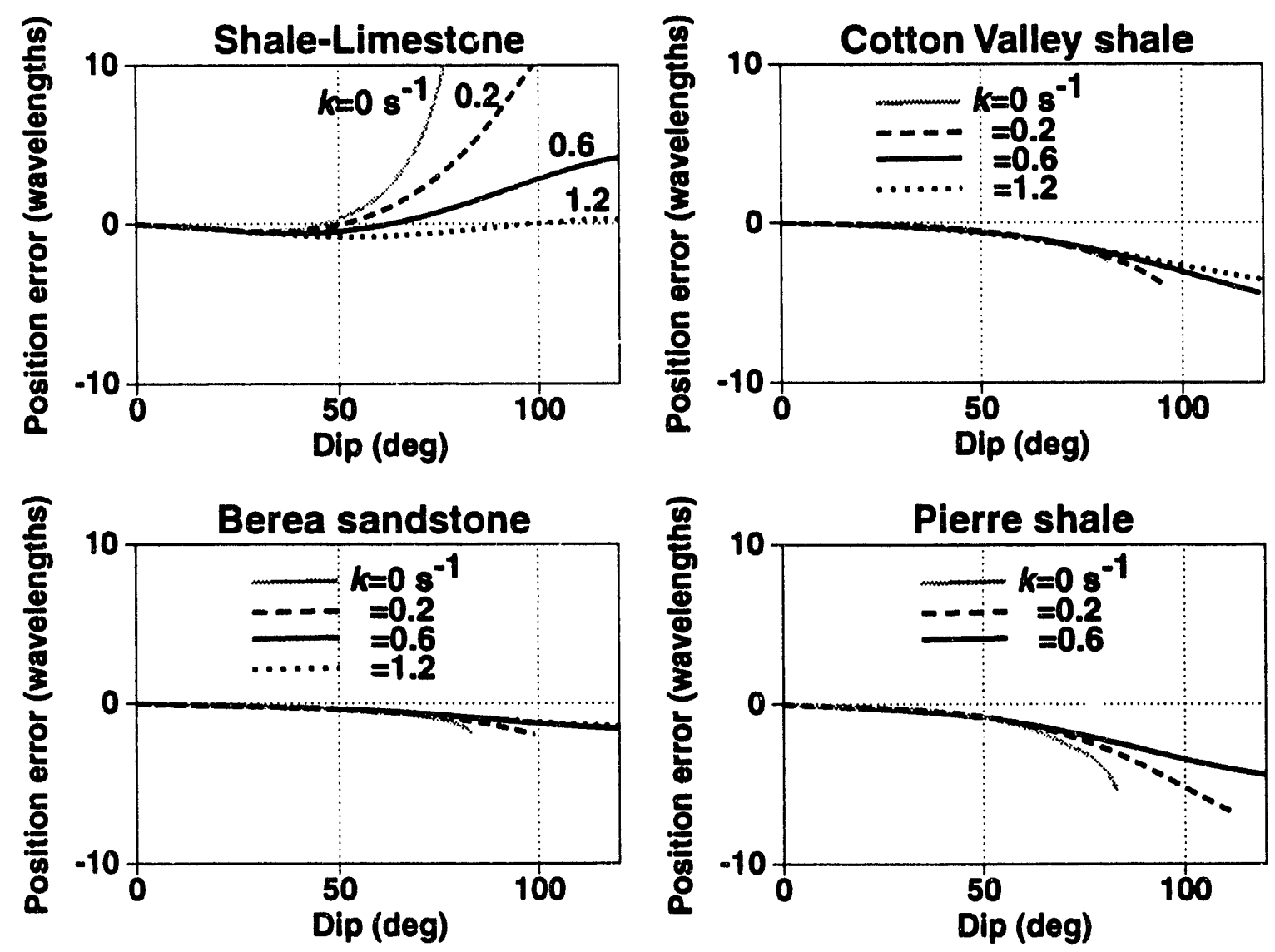

Fig. 11. Normalized position error (at $30 \mathrm{~Hz}$ ) as a function of reflector dip for the four media listed in Table 1 , with velocity gradients $k=0.0,0.2,0.6$, and $1.2 \mathrm{~s}^{-1}$. The reflector depth is $1500 \mathrm{~m}$ in all cases. 
turn close to horizontal. As often happens, intuition fails when it comes to anisotropy. The diametrically opposing behavior of errors for the shale-limestone and the Cotton Valley shale is particularly striking, again because the ratio of horizontal to vertical velocity, $V_{A} / V_{C}$, is almost identical in the two media.

Based on the migration errors in Figure 11, we would infer that the shale-limestone has the greatest degree of anisotropy, followed by the Pierre shale and Cotton Valley shale, and finally the Berea sandstone-not quite the order that would be predicted on the basis of $V_{A} / V_{C}$. Note that errors for the Cotton Valley shale are somewhat less dependent on the velocity gradient than are those for the Pierre shale (the curve for gradient $k=1.2 \mathrm{~s}^{-1}$ in Pierre shale is not shown).

While linear $\left.v_{(}^{\prime} z\right)$ is generally not expected for the earth's subsurface, to the extent that linear $v(z)$ holds, $k=0.6 \mathrm{~s}^{-1}$ is a somewhat representative value: $k=1.2 \mathrm{~s}^{-1}$ is on the high side, and $k=0.2 \mathrm{~s}^{-1}$ is clearly too small to yield the velocity increases with depth that are normally encountered. Thus, for all four media the "representative" case, $k=0.6 \mathrm{~s}^{-1}$, exhibits errors no larger than three or four wavelengths (and periods) even for dips as large as 120 degrees.

\section{Dependence on reflector depth and on stacking velocity}

Focusing our attention on the shale-limestone medium, Figure 12 skows the dependence of migration error on medium velocity and reflector depth. As throughout this paper, the ratios of the four velocity quantities characterizing the transverse isotropy are independent of depth and match those for the velocities listed in Table 1. In generating the three curves shown in the top portion of the figure, the vertical velocity at the surface $v_{0}$ was set so as to yield vertical average velocities of $3000 \mathrm{~m} / \mathrm{s}$, $2200 \mathrm{~m} / \mathrm{s}$, and $1500 \mathrm{~m} / \mathrm{s}$ at the reflector depth. For linear $v(z)$ media characterized by equation (1), the relationship between surface velocity $v_{0}$ and vertical average velocity $V_{a v g}(z)$ is given by

$$
V_{a v g}(z)=\frac{\int_{0}^{z} d \sigma}{\int_{0}^{z} \frac{d \sigma}{v_{0}+k \sigma}}=\frac{k z}{\log \left(1+\frac{k z}{v_{0}}\right)}
$$

So, given $k z$ and $V_{\text {avg }}$, we have

$$
v_{0}=\frac{k z}{\exp \left(\frac{k z}{V_{a v g}}\right)-1}
$$

In Figure 12, the solid black curve pertains to parameters that are close to those that generated the solid black curve in Figure 10. The dependence of errors on average velocity and on depth exhibit much similarity. The errors tend to become less positive with either increasing reflector depth or decreasing velocity. Inspection of Figure 12 suggests that the shape of an error curve is independent of either the depth or average velocity so long as the ratio of the two is held constant and the reference frequency $(30 \mathrm{~Hz}$ in all these tests) is fixed. Since this ratio is proportional to migrated reflection time, we infer that the shape and, indeed, the sign of the error curve for 

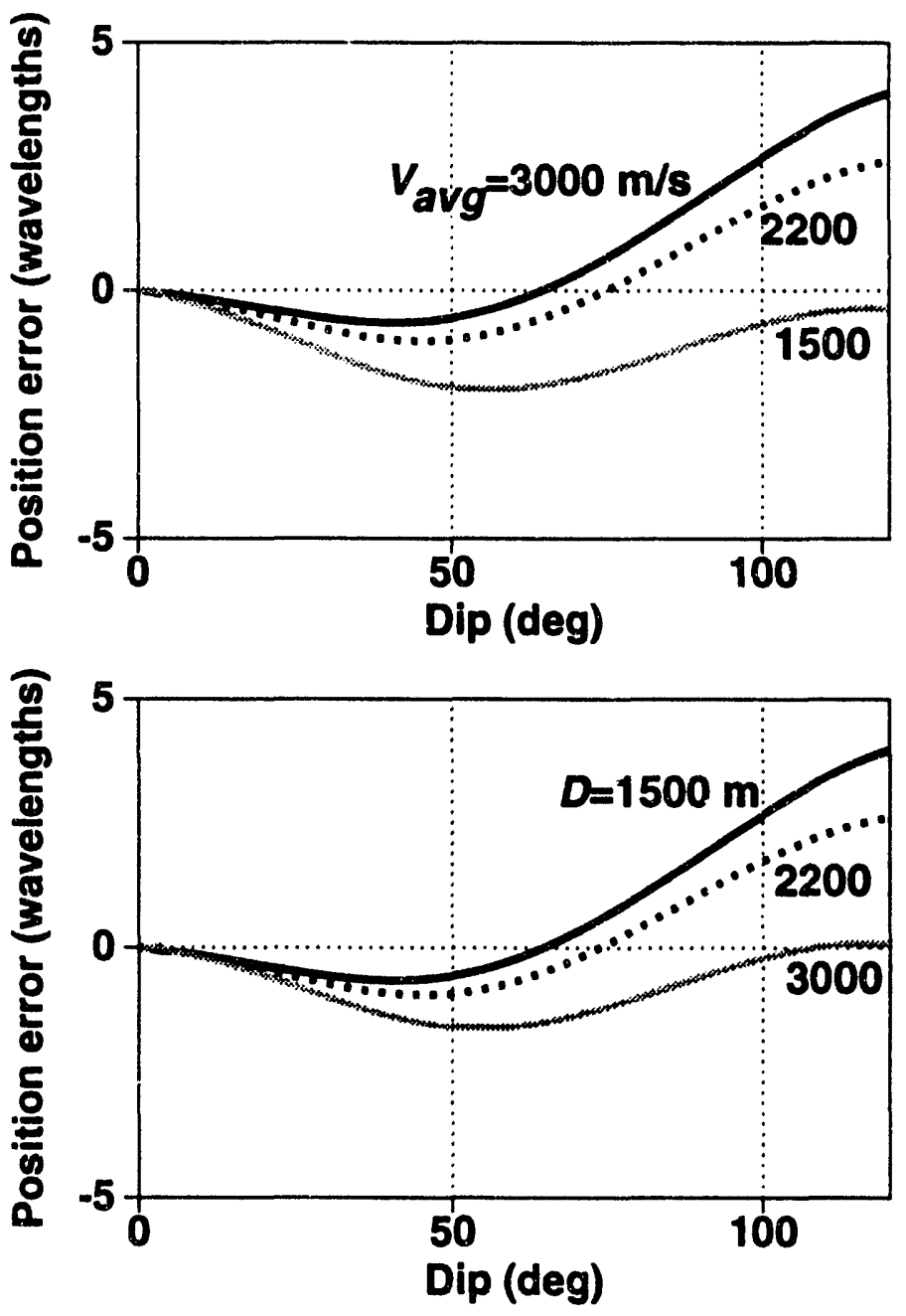

FIG. 12. Velocity dependence (top) and depth dependence (bottom) of normalized position errors as a function of reflector dip for FAI shale-limestone. All models have the "representative" velocity gradient, $k=0.6 \mathrm{~s}^{-1}$. Depth is held constant $(1500 \mathrm{~m})$ for the velocity tests on the top, and vertical average velocity is held constant $(3000 \mathrm{~m} / \mathrm{s})$ for the depth tests on the bottom. 
the shale-limestone is governed by the migrated reflection time. Thus depending on the migrated reflection time the data may be either over-migrated or under-migrated when anisotropy is not taken into account in the migration process. The Appendix summarizes a proof that, with the gradient $k$ and reference frequency held fixed, the normalized migration error indeed depends on reflector depth and average velocity only through their ratio, or equivalently, migrated reflection time.

Thus, inferences made in the preceding section about the relative importance of anisotropy for the four different media must be reviewed in the light that error behavior for any given medium can vary considerably with migrated reflection time. The complexity of the migration-error behavior for the different media emerges in Figure 13.
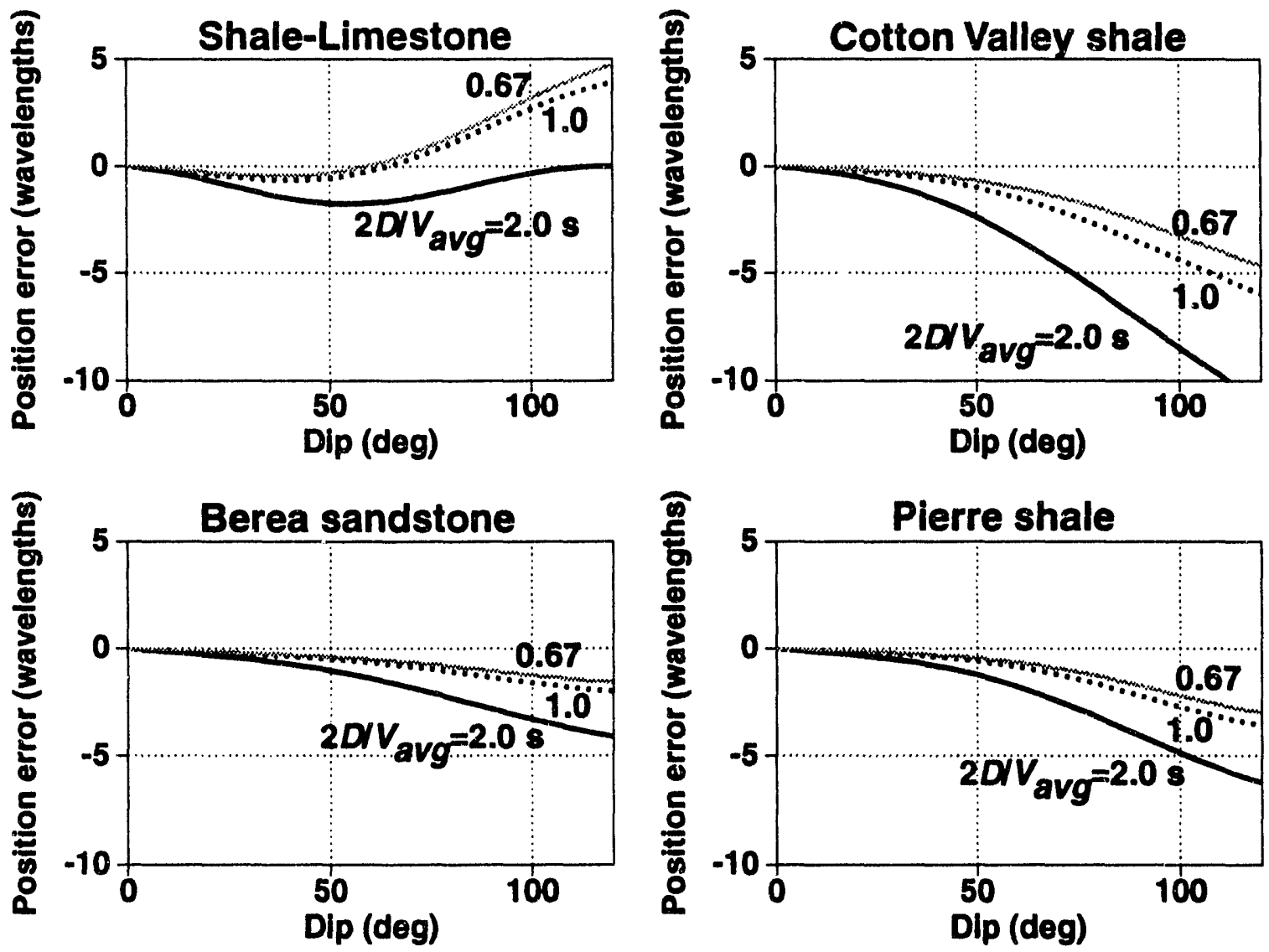

FIG. 13. Normalized position errors as a function of reflector dip for the four media listed in Table 1 , for three values of migrated reflection time $2 D / V_{\text {avg. }}$. All models have the same velocity gradient, $k=0.6 \mathrm{~s}^{-1}$.

For all the models treated in Figure 13, the velocity gradient is $0.6 \mathrm{~s}^{-1}$. The three error curves shown for each of the four media pertain to average velocity and reflector depth chosen so that the migrated reflection time is $0.67,1.0$, and $2.0 \mathrm{~s}$.

While it is satisfying to find that the migration errors depend only on migrated reflection time, that dependence, nevertheless, can be significant. For the shale- 
limestone medium, for example, ignoring anisotropy leads to under-migration for shallow reflectors, but to over-migration of deeper reflectors. Moreover, at migrated times later than about $1 \mathrm{~s}$, reflections in the Cotton Valley shale become significantly over-migrated. Even the "relatively isotropic" Berea sandstone exhibits a growing over-migration of the later reflections. If we were to rank degree of anisotropy on the basis of migration error for reflections at or later than $2 \mathrm{~s}$, migrated time, we would say that the Cotton Valley shale is the most anisotropic, followed by the forre shale, Berea sandstone, and then the shale-limestone- a different ordering than what we inferred above, when all the reflectors werc at the same depth and the vertical average velocities were given in Table 1.

In practice, the trend toward increasing normalized position error with increasing migrated reflection time, seen in Figure 13, is ameliorated by the tendency for dominant frequency to decrease with increasing reflection time. Thus, while unnormalized position (and time) errors certainly increase with increasing reflection migrated time, given the tendency for dominant frequency to decrease with increasing reflection time, the normalized errors should exhibit less dependence on migrated reflection time than that shown in Figure 13.

\section{DISCUSSION AND CONCLUSION}

Failure to take anisotropy into account in migration leads to position errors whose magnitude and sign both depend not only on the various elastic moduli of the subsurface medium, but also on migrated reflection time. These migration errors cannot at all be predicted on the basis of the ratio, $V_{A} / V_{C}$, of the horizontal to vertical velocity. (Recall that this ratio is nearly identical for the shale-limestone and Cotton Valley shale, which exhibit greatly differing error behavior in Figure 13.) In fact, any attempt to determine which of several media is "most" anisotropic and which is "least" is doomed to frustration. The answer is "it depends on the situation."

If, for example, our problem is one of converting from time to depth based on velocity analysis, then for an elliptically anisotropic medium, the measure of importance would be the ratio $V_{A} / V_{C}$. However, elliptical anistropy is a poor assumption for pwave behavior, and, as Thomsen (1986) has pointed out, this ratio has no influence whatsoever on the relationship between vertical velocity and the velocity estimated from conventional velocity analysis. Alternatively, if our problem is one of assessing migration error, then our ranking of degree of anisotropy might be based on size of error-and even then, the answer would depend on migrated reflection time.

Of course the correct thing to do is migrate with an algorithm that takes anisotropy into account. VerWest (1989) discussed migration in elliptically anisotropic media, and Uren, Gardner, and McDonald (1990) have shown results on model-tank data migrated with a frequency-wavenumber-domain approach that takes rather general anisotropy into account. However, it is not algorithmic limitations that prevent the use, in practice, of migration approaches that understand how to treat aniso- 
tropy. Rather, our information about the anisotropic characteristics of the subsurface is woefully inadequate. We have seen that the most readily accessible measure of anisotropy - the ratio of stacking velocity to vertical velocity-does not provide sufficient information about the pertinent elastic moduli. In fact, the elastic modulus $F$ is not at all obtainable from surface seismic data alone. While we do not have a good means of quantifying anisotropic behavior either routinely or otherwise in practice, studies suggest that anisotropy is the rule, and the degree of anisotropy is often considerable.

The limitations go deeper than this, however. Consider, for example, the many assumptions about the medium made for the analysis in this paper. The models studied are all (1) transversely isotropic, with (2) vertical axis of symmetry, with (3) velocity variation in depth only, with (4) constant gradient, and (5) all ratios among the four pertinent elastic moduli are independent of depth. While this combination of assumptions enabled efficient ray-trace calculation of the diffraction times required in the analysis, we can give little justification of the appropriateness of these models other than: (1) they provide more generality, and perhaps more realism, than do isotropic models and (2) there is little justification for models of anisotropy that differ substantially from those used here. (One possibile alternative to the FAI media considered here would be media in which, more true to observation, the ratio of vertical s-wave velocity to vertical p-wave velocity changes slowly wit' depth. Such media, however, may not lend itself to such straightforward ray tracing.)

Clearly, the studies here show that models of anisotropy are inadequate in describing migration error when inhomogeneity is not also taken into account. Also, while the subsurface is, of course, not a constant-gradient medium, the range of gradients studied here do provide examples of highly inhomogeneous media.

Ratcliff, et al. (1992) and Hale, et al. (1992) have presented examples from the Gulf of Mexico of stunning, apparently quite accurate, migrations of overhanging salt-dome flanks and of thin salt intrusions $(100-\mathrm{m}$ wide with more than $1-\mathrm{km}$ vertical relief) into faults surrounding salt domes. How could such features be imaged so well given that the migration algorithms did not take anisotropy into account? While the errors for some of the test cases here become sizeable for dips approaching and exceeding 90 degrees, results here also suggest that if the subsurface in the Gulf of Mexico is not "strongly anisotropic" in some appropriate sense, migration errors due to the combination of anisotropy and inhomogeneity may be no more than two or three wavelengths even for dips beyond 90 degrees. While two or three wavelengths is not insignificant to explorationists, the common practice of trial-and-error selection of migration velocity can readily accommodate errors of that size.

The error analysis in this study was limited to that for post-stack migration. Larner (1992) similarly treats characteristics of dip-moveout for this same type of anisotropic, inhomogeneous media. The extension of the methodology here to prestack time migration is the subject of a future paper. 
Final comment: While numerical estimation of migration error of the sort done here is no substitute for actual application of migration algorithms on synthetic and field data, such actual migration demonstrations would have been totally impractical and inadequate for attempts to understand the dependence of migration error on the range of model parameters considered here.

\section{ACKNOWLEDGMENTS}

We thank Dave Hale for his useful insights at critical stages of this study and Michel Dietrich for his critical review of the paper. Financial support for this work was provided in part by the United States Department of Energy, Grant Number DE-FG02-89ER14079 (this support does not constitute an endorsement by DOE of the views expressed in this paper) and by the members of the Consortium Project on Seismic Inverse Methods for Complex Structures at the Center for Wave Phenomena, Colorado School of Mines. 


\section{REFERENCES}

Byun, B., 1984, Seismic parameters for transversly isotropic media: Geophysics, 49, 1908-1914.

Červený V., 1989, Ray tracing in factorized anisotropic inhomogeneous media: Geophys. J. Int., 99, 91-100.

Gazdag, J., 1978, Wave equation migration with the phase shift method: Geophysics, 43, 1342-1351.

Hale, D., Hill. N. R., and Stefani, J. P., 1991, Imaging salt with turning waves: Soc. Explor. Geophys., 61st Ann. Intl. Mtg., Houston, 1171-1174.

Larner, K., 1992, Dip-moveout error in transversely isotropic media with linear velocity variation in depth: 62nd Annual Internat. Mtg., Soc. Expl. Geophys., Expanded Abstracts.

Levin, F. K., 1979, Seismic velocities in transversely isotropic media: Geophysics, 44, 918-936.

Levin, F. K., 1990, Reflection from a dipping plane-Transversely isotropic solid: Geophysics, 55, 851-855.

Ratcliff, D. W., Gray, S. H., and Whitmore Jr., N. D., 1992, Seismic imaging of salt structures in the Gulf of Mexico: The Leading Edge, 11, 15-31.

Schneider, W. A., 1978, Integral formulation for migration in two and three dimensions: Geophysics, 43, 49-76.

Seriff, A. J., 1986, Velocity anisotropy of seismic waves-illustrations: Geophys. Soc. of Houston Continuing Education Seminar, 1-25.

Shearer, P. M. and Chapman, C. H., 1988, Ray tracing in anisotropic media with linear velocity gradient: Geophys. J. Int., 94, 575-580.

Slotnick, M.M., 1986, Lessons in seismic computing: Society of Exploration Geophysicists, Tulsa, OK.

Thomsen, L., 1986, Weak elastic anisotropy: Geophysics, 51, 1954-1966.

Uren, N. F., Gardner, G. H. F., and McDonald, J. A., 1990, The migrator's equation for anisotropic media: Geophysics, 55, 1429-1434.

VerWest, B. J., 1989, Seismic migration in elliptically anisotropic media: Geophys. Prosp., 37, 149-166.

White, J. E., 1983, Underground sound: Application of sound waves: Elsevier, Amsterdam. 


\section{APPENDIX: \\ DEPENDENCE OF MIGRATION ERROR ON MIGRATED REFLECTION TIME}

Consider an FAI, transversely isotropic, linear $v(z)$ medium, defined by the ratios among the four pertinent elastic moduli and by the value of vertical velocity gradient $k$, as given in equation (1). Following Shearer and Chapman (1988), Larner (1992) shows that all raypaths within such a medium are simply scaled versions of one another, when represented in the $x_{1}, x_{3}$ coordinate system described in reference to Figure (4). Now suppose that a scatterer is located at depth $Z$ and that the average vertical velocity between the surface and the scatterer is $V_{\text {avg }}(Z)$, so that the migrated time $T_{m}$ (i.e., vertical time from the surface to scatterer) is given by

$$
T_{m}=\frac{2 Z}{V_{\text {avg }}(Z)} .
$$

For linear $v(z)$ media, equation (18) relates the average vertical velocity $V_{\text {avg }}(Z)$ to the velocity at the surface $v_{0}$ and to that at depth $Z, v(Z)$. Combining equations (18) and $(\mathrm{A}-1)$ gives

$$
\frac{v(Z)}{v_{0}}=\exp \left(\frac{k T_{m}}{2}\right) \text {. }
$$

Now, consider two different media with different velocities at the surface and with scatterers at different depths, but both with the same ratios among the elastic moduli, the same gradient $k$, and the same migrated time $T_{m}$ to the scatterer. Equation (A-2) shows that the ratio of velocity at the scatterer to velocity at the surface is the same for the two media. It then follows, from equation (1), that

$$
\frac{v(Z)-v_{0}}{v_{0}}=\frac{k Z}{v_{0}}
$$

is also the same for the two media. That is, $v_{0}$ is proportional to the scatterer depth $Z$. Also, from equation (A-2), the vertical velocity at the scatterer $v(Z)$ is likewise proportional to $Z$ between the two media. In fact, if the ratio of scatterer depths $Z_{2} / Z_{1}$ for the two media is $m$, it follows from equation (1) that for any pair of depths $z_{2} / z_{1}=m$ in the two media, the ratio of vertical velocities $v\left(z_{2}\right) / v\left(z_{1}\right)=m$, as well. Finally, this proportionality holds for the velocities in any direction through the FAI property that the ratios among the elastic moduli are independent of depth.

Thus, the two media described here are simply scaled versions of one another. Consequently, the shape of the raypath from a dipping reflector at depth $Z_{2}$ in medium 2 is identical to that from a reflector with the same dip at depth $Z_{1}$ in medium 1 . Being inversely proportional to velocity, the ray parameters for the two raypaths thus satisfy

$$
\frac{p_{1,2}}{p_{1,1}}=\frac{1}{m}=\frac{Z_{1}}{Z_{2}}
$$


Also, the lateral migration distances $\Delta y_{1}$ and $\Delta y_{2}$ for reflections from the two reflectors are in the same proportion as the scatterer depths in the two media. That is,

$$
\frac{\Delta y_{2}}{\Delta y_{1}}=\frac{Z_{2}}{Z_{1}} .
$$

This relationship holds not only for the correct migration distances, but also for the erroneous migration distances that arise when isotropy is assumed for the migration (isotropy linear $v(z)$ media is a special case of the FAI media considered here). Therefore the differences between the true and erroneous migration distances, the position errors, must be in the same proportion.

Finally, if we are considering the same reference frequency for computation of the normalized position errors in the two media, it follows from equation (14) and equation (A-3) that the normalized position errors are the same for the two media. 

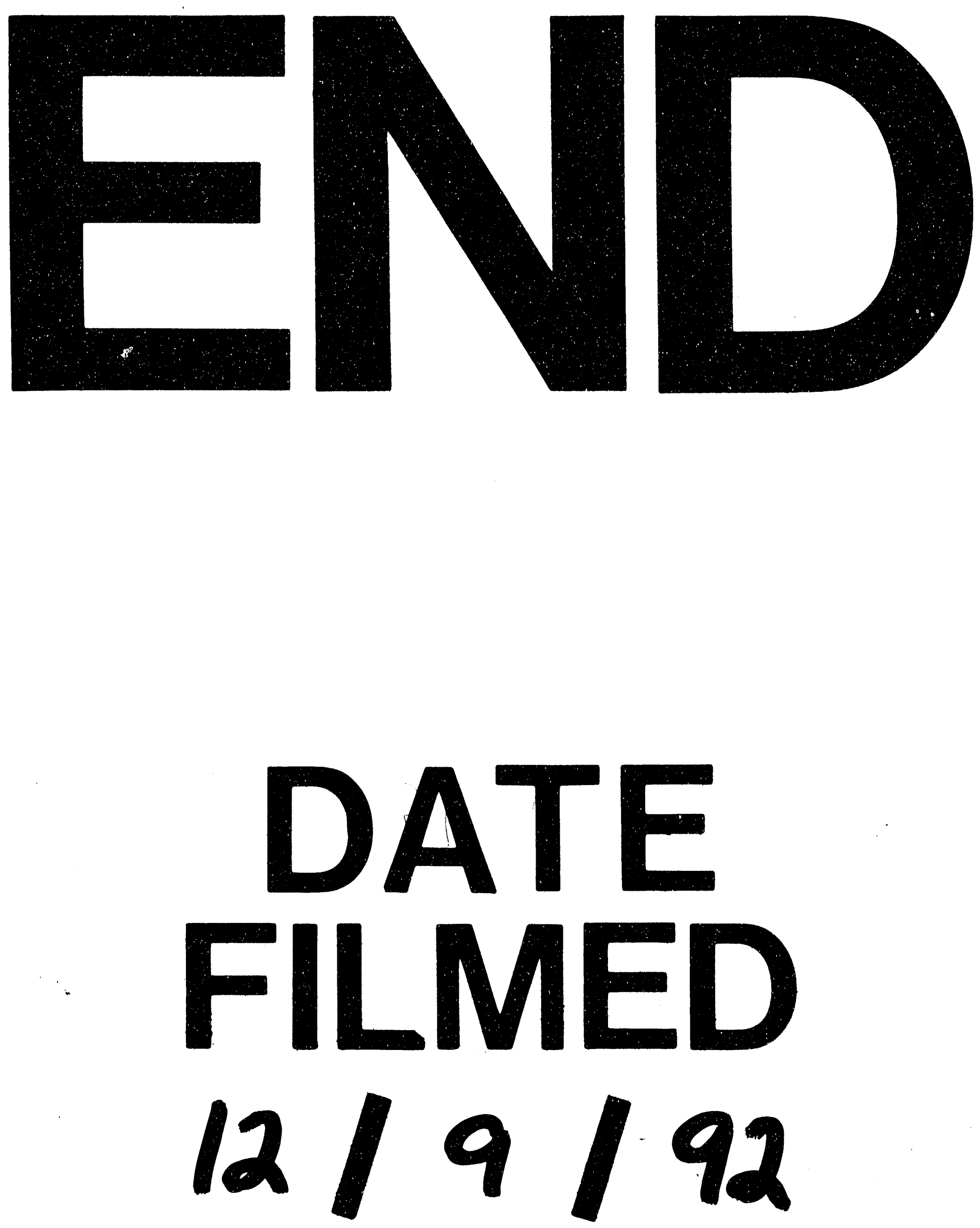

I 
